\title{
Performance evaluation of multicast MISO-OFDM systems
}

\author{
Berna Özbek · Didier Le Ruyet · Hajer Khanfir
}

Received: 3 September 2007 / Accepted: 11 March 2008 / Published online: 26 April 2008

(C) Institut TELECOM and Springer-Verlag France 2008

\begin{abstract}
In this paper, we analyze the performance of multicast orthogonal frequency division multiplexing (OFDM) systems with single and multiple transmit antennas. We show that the resource allocation that includes the subcarrier allocation, bit loading, and the precoding vector selection in the multiple-input singleoutput (MISO) case is a difficult optimization problem. Consequently, we propose suboptimal algorithms based on the maximization of the sum data rate and the maximization of the minimum user data rate criteria. For practical application, we consider a complete transmission chain by combining powerful erasure codes with the proposed algorithms. Using this scheme, we guarantee that each user receives the same amount of information to decode the same data. Simulation results show that, for both single-input single-output (SISO)-OFDM and MISO-OFDM cases, the proposed multicast OFDM systems achieve gains over the worst user case algorithm.
\end{abstract}

The work of Berna Özbek was supported by the FP6-IYTE wireless project.

The work of Didier Le Ruyet and Hajer Khanfir was supported by the Euripides European project SMART.

\section{B. Özbek $(\bowtie)$}

Electrical and Electronics Engineering Department, Izmir Institute of Technology, Urla 35430, Izmir, Turkey

e-mail: bernaozbek@iyte.edu.tr

D. Le Ruyet · H. Khanfir

Electronics and Communications Laboratory,

Conservatoire National des Arts et Métiers (CNAM),

292 rue Saint Martin, 75141, Paris, France

D. Le Ruyet

e-mail: leruyet@cnam.fr
Keywords Multicast OFDM - Adaptive resource allocation • Multiple transmit antennas • Precoding vector optimization . Subcarrier and bit allocation $\cdot$ Erasure codes

\section{Introduction}

Next-generation wireless communication systems will provide a wide range of applications with high and time-varying bandwidth requirements. Up to now, the main wireless applications are broadcasting multiuser systems. However, the demand for multimedia services, such as video and audio conferencing, online training, news and software distribution, and database replication, is increasing. For these applications, multicasting offers a significant improvement compared to broadcasting because it allows the transmission of packets to multiple destinations using fewer resources [1]. However, since in the wireless channel the received signalto-noise ratio of each user is not the same, the data rate of the multicast stream can be limited by the data rate of the least capable user. In this case, this method cannot provide efficient performance when the number of users in the group increases.

The main difficulty in achieving a high data rate on the wireless channels is known to be the frequency selectivity and the fading due to the existence of multiple paths. Orthogonal frequency division multiplexing (OFDM) techniques can significantly alleviate the impacts of frequency selective fading [2] and are attractive for the next generation of wireless systems. Moreover, multiple-input single-output (MISO) systems that use multiple transmit antenna techniques [3] can be 
combined with OFDM systems [4] to enhance the performance of wireless systems in fading channels.

In $[5,6]$, by assuming that the transmitter knows the channel state information (CSI) of all the users, it has been shown that adaptive subcarrier and bit allocation can significantly increase the data rate of broadcasting multiuser OFDM systems. Recently, it has been demonstrated in [7] that using OFDM with subcarrier and bit allocation, it is also possible to increase the data rate on each subcarrier for multicasting applications by selecting only the users with a good channel condition.

In this paper, we propose suboptimal adaptive resource allocation algorithms to improve the performance of multicast OFDM systems with both single and multiple transmit antennas. Firstly, for multicast single-input single-output (SISO)-OFDM systems, we show that the allocation of the subcarriers to the users and loading bits to the subcarriers is an optimization problem. We solve this optimization problem with a suboptimal low-complexity algorithm. Our purpose is to minimize the transmission time by maximizing the minimum user data rate. Then, we extend the proposed algorithm to the multiple transmit antennas case. For multicast MISO-OFDM systems, similar to the SISOOFDM case, the determination of the precoding vector and the allocation of the subcarriers to the users and loading bits to the subcarriers is an optimization problem. We solve this optimization problem with a suboptimal low-complexity algorithm [8] by maximizing the minimum user data rate instead of adapting the rate according to the worst user. For practical applications, we propose a complete transmission chain where each user will be able to decode the same data information using powerful erasure codes [9].

Instead of using automatic repeat request (ARQ), the proposed transmission chain is based on erasure codes. Indeed, it is well known that the ARQ strategy can quickly overwhelm the receiver request for the retransmission of lost packets. A solution to this problem is to use the fountain concept using erasure codes such as Luby transform (LT) [10], digital fountain codes [11], or Raptor codes [12]. The basic principle behind the use of erasure codes is that the original source data are encoded in the form of a sequence of packets. These packets are transmitted and the users can reconstruct the original source data once they receive a sufficient number of encoded packets. The main benefit of this approach is that different receivers can recover the source data using different encoded packets.

This paper is organized as follows: First, we describe the system model of multicast OFDM systems with both single and multiple transmit antennas over wireless channels in Section 2. We present the adaptive resource allocation algorithms for SISO and MISOOFDM systems, and then we propose a low-complexity suboptimal algorithm in Section 3. In Section 4, we propose a complete transmission chain for practical multicast SISO-OFDM applications, and then extend it to the MISO-OFDM with the proposed algorithm. Finally, in Section 5, we present the simulation and comparison results by evaluating the user data rate supported by the transmission chain and show the percentage of power saving.

\section{System model for multicast OFDM systems}

In this section, we will consider multicast OFDM systems with both single and multiple transmit antennas. The base station will serve $K$ users with only one receive antenna. The common data are formed into OFDM symbol with $N$ subcarriers and then transmitted through frequency selective channels. These channels are assumed to be constant over one frame that includes $P_{f}$ OFDM symbols and varying between the frames considering the Doppler frequency. Moreover, it is assumed that the number of channel taps is equal to or smaller than the length of the guard interval to avoid intercarrier and intersymbol interference at the receiver.

Assuming that the channel information about all the subcarriers for all the users are known at the transmitter, the adaptive resource allocation algorithm is used to optimize the system parameters in a way that maximizes the total number of bits received by all the users. Each subcarrier is assigned to a group of users that receive the same data, and then the number of bits on each subcarrier is determined considering the lowest channel gains among the users allocated to this subcarrier. It is assumed that the subcarrier/bit allocation information is transmitted to each user through a separate control channel.

Before defining the optimization problem, we will first give the notations for the $p$ th frame. For the sake of clarity, we will derive the equations assuming that one frame is only composed of one OFDM symbol $\left(P_{f}=1\right)$. Let $R_{p, k}$ be the data rate of the $k$ th user and $c_{p, n}$ be the number of bits that are assigned to the $n$th subcarrier. Here, the user index is unnecessary because the users using the same subcarrier receive identical data using the same modulation. $c_{p, n}$ is selected from the set of $\{0,1,2, \ldots, M\}$, where $M$ is the maximum number of bits per symbol that can be transmitted by each subcarrier $n=1,2, \ldots, N$. 
At each frame, the user data rate and the sum data rate are calculated, respectively, as

$R_{p, k}=\sum_{n=1}^{N} c_{p, n} \rho_{p, k, n}$

$R_{p}=\sum_{k=1}^{K} R_{p, k}$

where $\rho_{p, k, n}$ is a binary value indicating whether the $k$ th user utilizes the $n$th subcarrier or not.

$\rho_{p, k, n}= \begin{cases}1 & \text { if the } n \text {th subcarrier is used for } k \text { th user } \\ 0 & \text { else }\end{cases}$

\subsection{SISO case}

The multicast OFDM system with single transmit antenna is shown in Fig. 1. In this system, the channel between the base station and the $k$ th user is described in the frequency domain as

$\mathbf{H}_{p, k}=\left[\begin{array}{llll}H_{p, k, 1} & H_{p, k, 2} & \ldots & H_{p, k, N}\end{array}\right]^{T}$

where $H_{p, k, n}$ is the channel gain from the transmitter to the $k$ th user for $n$th subcarrier. In this scheme, the received signal for the $k$ th user and $n$th subcarrier is written as

$Y_{p, k, n}=H_{p, k, n} S_{p, n}+N_{p, k, n}$

where $S_{p, n}$ is the common transmitted symbol at the $n$th subcarrier and $N_{p, k, n}$ is the noise at the $k$ th user and $n$th subcarrier.

Assuming that the available total transmit power (in energy per symbol) is limited by $P_{T}$, to maximize

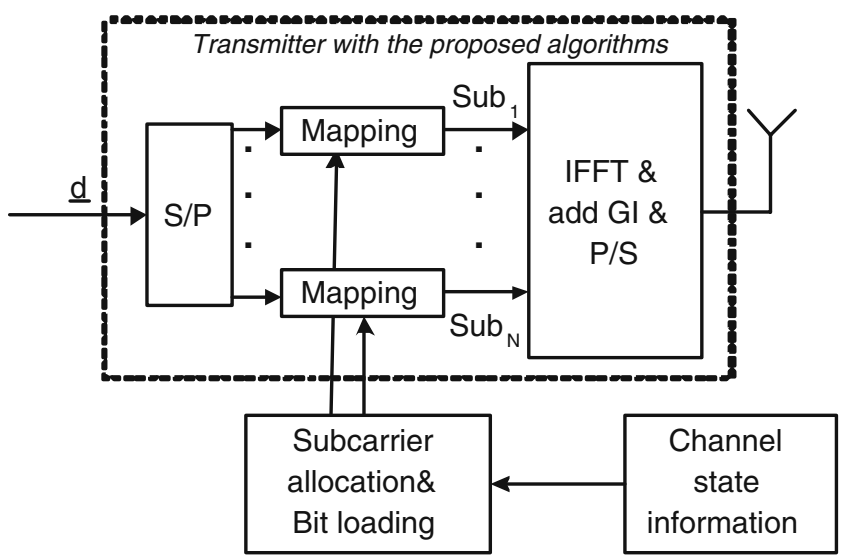

Fig. 1 The transmitter structure for multicast SISO-OFDM system the sum data rate, the optimization problem can be expressed as

$\max _{c_{p} ; \rho_{p}} R_{p}=\max _{c_{p} ; \rho_{p}} \sum_{k=1}^{K} \sum_{n=1}^{N} c_{p, n} \rho_{p, k, n}$

subject to

$\sum_{n=1}^{N} \max _{k}\left(\frac{f\left(c_{p, n}\right) \rho_{p, k, n}}{\left|H_{p, k, n}\right|^{2}}\right) \leq P_{T}$

where $f\left(c_{p, n}\right)$ is the required received power (in energy per symbol) for a reliable reception of $c_{p, n}$ bits when the channel gain is equal to unity.

Besides that, to maximize the minimum user data rate, the optimization problem can be written by

$\max _{c_{p} ; \rho_{p}} \min _{k} R_{p, k}=\max _{c_{p} ; \rho_{p}} \min _{k} \sum_{n=1}^{N} c_{p, n} \rho_{p, k, n}$

subject to

$\sum_{n=1}^{N} \max _{k}\left(\frac{f\left(c_{p, n}\right) \rho_{p, k, n}}{\left|H_{p, k, n}\right|^{2}}\right) \leq P_{T}$

For M-ary quadrature amplitude modulation (M-QAM) schemes, the bit error probability is upper bounded by the symbol error probability, which is tightly approximated by $4 Q\left(\sqrt{d^{2} /\left(2 N_{0}\right)}\right)$ [13] where $d$ is the minimum distance between the points in the signal constellation. Because the average energy of a M-QAM symbol is equal to $(M-1) d^{2} / 6$, the required power (in energy per symbol) $f\left(c_{p, n}\right)$ for supporting $c_{p, n}$ bits per symbol at a required bit-error-rate (BER) $p_{e}$ can be represented by

$f\left(c_{p, n}\right)=\frac{N_{0}}{3}\left[Q^{-1}\left(p_{e} / 4\right)\right]^{2}\left(2^{c_{p, n}}-1\right)$

where $N_{0} / 2$ denotes the variance of the additive white Gaussian noise and $Q(x)=\frac{1}{\sqrt{2 \pi}} \int_{x}^{\infty} \mathrm{e}^{-t^{2} / 2} d t$.

\subsection{MISO case}

The multicast OFDM system with $N_{t}$ transmit antennas is shown in Fig. 2. In this system, the vector channel between the transmitter and the $k$ th user is described in the frequency domain by

$\mathbf{H}_{p, k, n}=\left[\begin{array}{lllll}H_{p, k, n, 1} & H_{p, k, n, 2} & \ldots & H_{p, k, n, N_{t}}\end{array}\right]^{T}$

where $H_{p, k, n, t}$ is the channel gain from the $t$ th transmit antenna to the $k$ th user for $n$th subcarrier. In this 
Fig. 2 The transmitter structure for multicast MISO-OFDM system

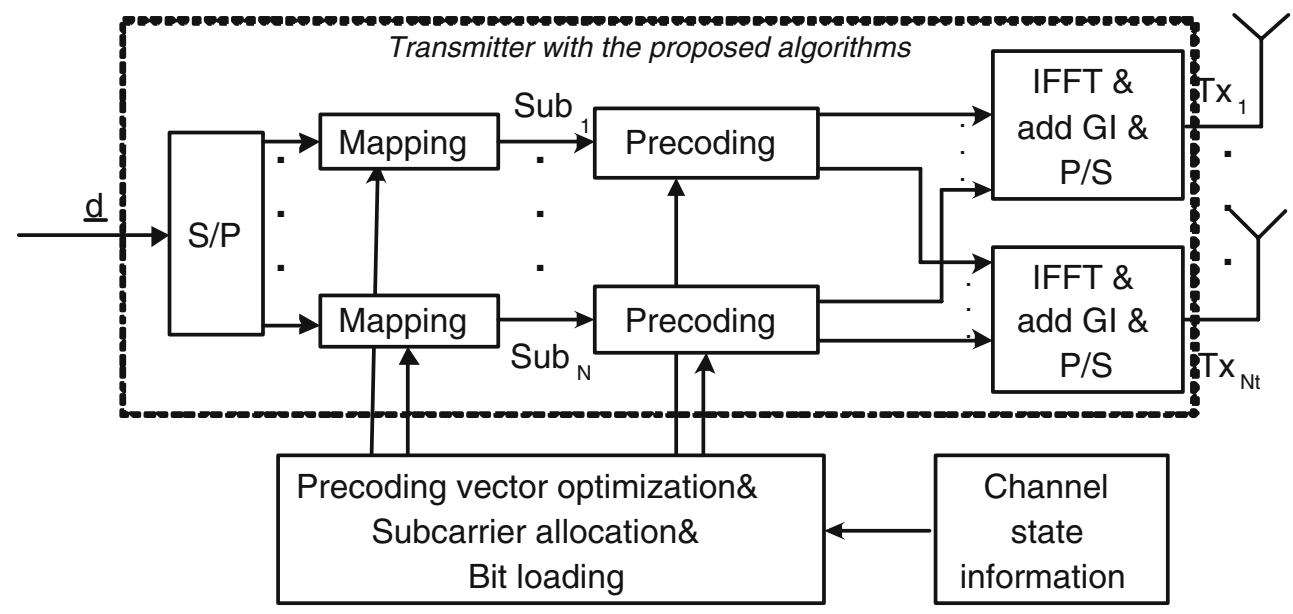

scheme, the received signal for the $k$ th user and the $n$th subcarrier is represented by

$Y_{p, k, n}=\mathbf{H}_{p, k, n} \mathbf{X}_{p, n}+N_{p, k, n}$

where $\mathbf{X}_{p, n}=\mathbf{W}_{p, n} S_{p, n}$ is the common precoded transmitted symbol at the $n$th subcarrier and $\mathbf{W}_{p, n}$ is the precoding vector of size $1 \times N_{t}$.

To maximize the sum data rate, the optimization problem can be given as

$\max _{\mathbf{W}_{p} ; c_{p} ; \rho_{p}} R_{p}=\max _{\mathbf{W}_{p} ; c_{p} ; \rho_{p}} \sum_{k=1}^{K} \sum_{n=1}^{N} c_{p, n} \rho_{p, k, n}$

subject to

$\sum_{n=1}^{N} \max _{k}\left(\frac{f\left(c_{p, n}\right) \rho_{p, k, n}}{\left|\mathbf{W}_{p} \mathbf{H}_{p, k, n}\right|^{2}}\right) \leq P_{T}$

To maximize the minimum user data rate, the optimization problem can be expressed as

$\max _{\mathbf{W}_{p} ; c_{p} ; \rho_{p}} \min _{k} R_{p, k}=\max _{\mathbf{W}_{p} ; c_{p} ; \rho_{p}} \min _{k} \sum_{n=1}^{N} c_{p, n} \rho_{p, k, n}$

subject to

$\sum_{n=1}^{N} \max _{k}\left(\frac{f\left(c_{p, n}\right) \rho_{p, k, n}}{\left|\mathbf{W}_{p} \mathbf{H}_{p, k, n}\right|^{2}}\right) \leq P_{T}$

\section{Adaptive resource allocation algorithms}

\subsection{SISO-worst-user algorithm}

In this algorithm, each user receives the transmitted information according to the worst user data rate for each subcarrier and, consequently, there is no optimization to perform. This algorithm is based on the maximization of the user data rate but is strongly suboptimal.
At frame $p$ :

For each subcarrier $n$ :

Step 1sw) Select worst user:

$\omega_{n}=\arg \min _{k}\left|H_{p, k, n}\right|$

End

Step 2sw) Bit loading (the modified Levin-Campello algorithm [5, 7]):

Let $\Delta P_{p, n}(c)=(f(c+1)-f(c)) /\left(u_{p, n}\left|H_{p, \omega_{n}, n}\right|^{2}\right)$ denotes the incremental power needed for the transmission of one additional bit at the subcarrier $n, c$ is the number of loaded bits for the $n$th subcarrier, and $u_{p, n}=\sum_{k=1}^{K} \rho_{p, k, n}$ is the number of users who share the $n$th subcarrier, which is necessary because the incremental power is shared by the group of users allocated to the subcarrier.

Let initializes the parameters as $c_{p, n}=0$ and $P_{T}^{*}=0$, where $P_{T}^{*}$ is tentative transmit power, then evaluates $\Delta P_{p, n}(0)$ for all $n$. Repeat the following iterations unless $P_{T}^{*} \geq P_{T}$

$$
\begin{aligned}
n^{*} & =\arg \min _{n} \Delta P_{p, n}\left(c_{p, n}\right) \\
P_{T}^{*} & =P_{T}^{*}+\Delta P_{p, n^{*}}\left(c_{p, n^{*}}\right) u_{p, n^{*}} \\
c_{p, n^{*}} & =c_{p, n^{*}}+1 \\
\text { if } c_{p, n^{*}} & =\mathrm{M} \text {, set } \Delta P_{p, n^{*}}\left(c_{p, n^{*}}\right) \\
& =\infty \text { else evaluate } \Delta P_{p, n^{*}}\left(c_{p, n^{*}}\right) .
\end{aligned}
$$

End

\subsection{SISO-two-steps algorithm}

For the single transmit antenna case, the problem of optimization includes the subcarrier allocation and bit 
loading. We consider a suboptimal approach where the bit allocation and the subcarrier allocation, assuming equal power for each subcarrier, are separated [7]. This algorithm is developed to maximize the sum data rate given in Eqs. 6 and 7 instead of maximizing the minimum user data rate.

At frame $p$ :

For each subcarrier $n$ :

\section{Step 1st) Subcarrier allocation:}

(a) Determine the number of bits allocated: Let $c_{p, k, n}$ be the number of bits that can be received by the $k$ th user in the case of $\rho_{p, k, n}=1$. The number of supportable bits is given by

$$
c_{p, k, n}=\min \left(f^{-1}\left(\frac{P_{T}}{N}\left|H_{p, k, n}\right|^{2}\right), M\right)
$$

(b) Select the user that maximizes the sum data rate:

Calculate the tentative sum data rate $\bar{R}_{p, k, n}$ when the $k$ th user is selected as the user requiring the maximum power.

$$
\bar{R}_{p, k, n}=u_{p, k, n} c_{p, k, n}
$$

where $u_{p, k, n}$ indicates the number of users who have channel gains larger than $H_{p, k, n}$. Select the user index $\kappa_{n}$ maximizing $\bar{R}_{p, k, n}$.

$\kappa_{n}=\arg \max _{k} \bar{R}_{p, k, n}$

(c) Allocate the users to the subcarrier:

$$
\rho_{p, k, n}= \begin{cases}1 & \text { if } \quad\left|H_{p, k, n}\right| \geq\left|H_{p, \kappa_{n}, n}\right| \\ 0 & \text { else }\end{cases}
$$

\section{End}

Step 2st) Bit loading:

The same algorithm as that given in Step 2sw by using $\mathrm{H}_{p, \kappa_{n}, n}$ instead of $\mathrm{H}_{p, \omega_{n}, n}$.

End

\subsection{Proposed SISO-three-steps algorithm}

In this algorithm, as in the SISO-two-steps algorithm, we first allocate the users by maximizing the sum data rate. Then, to increase the minimum user data rate, we propose to modify the subcarrier allocation at each frame considering the worst user data rate.
At frame $p$ :

Step 1sp) Subcarrier allocation:

The same algorithm as given in Step 1st.

Step 2sp) Modify the subcarrier allocation:

Initialize the tentative user data rate as, Set iterative index $i=0$ :

$\bar{R}_{p, k}^{(0)}=\sum_{n=1}^{N} c_{p, \kappa_{n}, n} \rho_{p, k, n}$

(a) Find the user that has the minimum user data rate:

$\beta=\arg \min _{k} \bar{R}_{p, k}^{(i)}$

(b) Find the subcarriers allowing to maximize the minimum user data rate:

Firstly, create a set $\mathbf{s}$ of the possible subcarriers allowing an increase at the data rate of the user $\beta$, which has the minimum user data rate:

$$
\begin{aligned}
\mathbf{s}=\{ & n_{i} \in\{1: N\} \mid c_{p, \beta, n_{i}} \\
& \left.-c_{p, \kappa_{n}, n_{i}} \rho_{p, \beta, n_{i}}>0\right\}
\end{aligned}
$$

(c) In this subcarrier set, find the subcarrier index $j$ that will maximize the minimum user data rate in the user set that does not include user $\beta$ :

$$
\begin{aligned}
j=\arg \max _{n_{i}} \min _{k \neq \beta}\left\{\bar{R}_{p, k}^{(i)}+c_{p, k, n_{i}} \rho_{p, k, n_{i}}^{\prime}\right. \\
\left.-c_{p, k_{n}, n_{i}} \rho_{p, k, n_{i}}\right\}
\end{aligned}
$$

where $\rho_{p, k, n}^{\prime}=1$ if the $k$ th user utilized at the subcarrier $n$ when $\beta$ is set as user index.

(d) Modify the subcarrier allocation at subcarrier $j$ :

$\kappa_{j}=\beta$

(e) Then calculate the tentative user data rate:

$R_{p, k}^{(i+1)}=R_{p, k}^{(i)}+c_{p, \beta, j} \rho_{p, k, j}^{\prime}-c_{p, \kappa_{n}, j} \rho_{p, k, j}$

(f) Continue until $\min _{k} \bar{R}_{p, k}^{(i+1)} \leq \min _{k} \bar{R}_{p, k}^{(i)}$. $i=i+1$. 
(g) The subcarriers reallocation:

For each subcarrier $n$ :

$$
\rho_{p, k, n}= \begin{cases}1 & \text { if }\left|H_{p, k, n}\right| \geq\left|H_{p, \kappa_{n}, n}\right| \\ 0 & \text { else }\end{cases}
$$

End

Step 3sp) Bit loading:

The same algorithm as given in Step $2 s w$ by using $\mathrm{H}_{p, \kappa_{n}, n}$ instead of $\mathrm{H}_{p, \omega_{n}, n}$.

\subsection{MISO-optimized-worst-user algorithm}

In this algorithm, the precoding vector is optimized using the max-min algorithm [14], and then the gain for each user is calculated. The transmission gain is defined according to the optimized-worst-user gain.

At frame $p$ :

For each subcarrier $n$ :

Step 1mwo) Optimize the precoding vector and evaluate the gain:

(a) Optimization of the precoding vector (the max-min algorithm):

$$
\max _{\mathbf{W}_{p, n}} \min \left\{\mathbf{H}_{p, n}^{H} \mathbf{W}_{p, n}^{H} \mathbf{W}_{p, n} \mathbf{H}_{p, n}\right\}
$$

subject to

$$
\mathbf{W}_{p, n} \mathbf{W}_{p, n}^{H} \leqslant 1
$$

where $\quad \mathbf{H}_{p, n}=\left[\mathbf{H}_{p, 1, n} \mathbf{H}_{p, 2, n} \ldots\right.$ $\left.\mathbf{H}_{p, K, n}\right]$.

(b) Calculate the gains according to the optimized precoding vector.

$\mathrm{G}_{p, n, k}^{\prime}=\left|\mathbf{W}_{p, n}^{\prime} \mathbf{H}_{p, k, n}\right|^{2}$

(c) Select the user that has the minimum gain.

$$
\omega_{n}^{\prime}=\arg \min _{k} \mathrm{G}_{p, k, n}^{\prime}
$$

\section{End}

Step 2mwo) Bit loading: The same algorithm as that given in Step $2 s w$ by using $\mathrm{G}_{p, \omega_{n}^{\prime}, n}^{\prime}$ instead of $\mathrm{H}_{p, \omega_{n}, n}$.

End

\subsection{Proposed MISO-two-steps algorithm}

The adaptive resource allocation includes the precoding vector optimization, subcarrier allocation, and bit loading. Because it is very difficult to solve Eq. 13 subject to Eq. 14, we propose to separate the bit allocation problem and solve both the precoding vector optimization and subcarrier allocation by assuming equal power for each subcarrier to maximize the sum data rate at each subcarrier. The expression of the new suboptimal optimization can be written as follows:

For each subcarrier $n$ :

$\max _{\mathbf{W}_{p, n} ; c_{p, n} ; \rho_{p, n}} \sum_{k=1}^{K} c_{p, n} \rho_{p, k, n}$

subject to

$\max _{k}\left(\frac{f\left(c_{p, n}\right) \rho_{p, k, n}}{\left|\mathbf{W}_{p, n} \mathbf{H}_{p, k, n}\right|^{2}}\right) \leq \frac{P_{T}}{N}$

This optimization problem can be solved for each subcarrier $n$ by considering all the $2^{K}-1$ possible user allocations and by searching the best precoding vector associated to each case.

Let $\boldsymbol{\rho}_{p, 1, n}, \boldsymbol{\rho}_{p, 2, n}, \ldots, \boldsymbol{\rho}_{p, i, n}, \ldots, \boldsymbol{\rho}_{p, 2^{K}-1, n}$ be the set of the non-zero vectors of the $K$ dimensional hypercube as follows:

$\rho_{p, 1, n}=(1,0, \ldots, 0), \rho_{p, 2, n}=(0,1, \ldots, 0), \ldots$,

$\rho_{p, K, n}=(0,0, \ldots, 1), \ldots, \rho_{p, 2^{K}-1, n}=(1,1, \ldots, 1)$

At each frame $p$ :

For each subcarrier $n$ :

For each index $i$ :

Step 1mpc) Subcarrier allocation and precoding vector selection:

(a) Optimization of the precoding vector (max-min algorithm):

$$
\begin{array}{r}
\max _{\mathbf{W}_{p, i, n}} \min \left\{\mathbf{H}_{p, k^{\prime}, n}^{H} \mathbf{W}_{p, i, n}^{H} \mathbf{W}_{p, i, n}\right. \\
\left.\times \mathbf{H}_{p, k^{\prime}, n} ; k^{\prime} \in \mathcal{K}\right\}
\end{array}
$$

subject to

$\mathbf{W}_{p, i, n} \mathbf{W}_{p, i, n}^{H} \leqslant 1$

where $\mathcal{K}=\left\{k \mid \rho_{p, i, n, k}=1\right\}$

(b) Calculation of the gains according to optimized precoding vector.

$\mathrm{G}_{p, i, n, k^{\prime}}^{\prime}=\left\|\mathbf{W}_{p, i, n}^{\prime} \mathbf{H}_{p, k^{\prime}, n}\right\|^{2}$

where $k^{\prime} \in \mathcal{K}$. 
(c) Determination of the number of bits allocated and the sum data rate:

$$
\begin{aligned}
& c_{p, i, n}= \min \left(f ^ { - 1 } \left(\frac{P_{T}}{N}\right.\right. \\
&\left.\left.\times \min _{k^{\prime}}\left(\mathrm{G}_{p, i, n, k^{\prime}}\right)\right), M\right) \\
& \bar{R}_{p, i, n}=\left(\sum_{k=1}^{K} \rho_{p, i, n, k^{\prime}}\right) c_{p, i, n}
\end{aligned}
$$

End

(d) Selection of the index that maximizes the sum data rate and the precoding vector:

$$
\begin{aligned}
& \kappa=\arg \max _{i} \bar{R}_{p, i, n} \\
& \mathbf{W}_{p, n}=\mathbf{W}_{p, \kappa, n}^{\prime}
\end{aligned}
$$

(e) Allocation of the users to the subcarrier:

$$
\rho_{p, k, n}=\rho_{p, \kappa, n, k^{\prime}}
$$

End

Step 2mpc) Bit Loading:

The bit loading algorithm is the same as Step $2 s w$ except that $H_{p, w_{n}, n}$ is replaced by $\mathrm{G}_{p, \kappa, n, k^{\prime}}^{\prime}$.

\subsection{Proposed low complexity MISO-two-steps algorithm}

Because the max-min algorithm is NP-hard, the problem can only be solved approximately. Following [14], using relaxation, the problem is transformed into a suitable form for semidefinite programming. Then, using the interior point method, the optimization is performed. Except for small values of $K$, this optimization problem is intractable. As a consequence, we propose a suboptimal algorithm to optimize the precoding vector and to choose the configuration of user allocation.

In order to avoid the complex optimization process, we propose to choose the matched filter (MF) precoding vectors among a limited set of precoding vectors. This set is composed of the normalized transpose conjugate of the channel vectors associated to each user. The selected precoding vector and user allocation maximize the sum data rate.
At each frame $p$ :

For each subcarrier $n$ :

Step $1 \mathrm{mp}$ ) Subcarrier allocation and precoding vector selection:

(a) Initializing of the precoding vector:

$$
\mathrm{W}_{p, i, n}^{\mathrm{init}}=\frac{\mathrm{H}_{p, i, n}^{H}}{\sqrt{\operatorname{tr}\left(\mathrm{H}_{p, i, n}^{H} \mathrm{H}_{p, i, n}\right)}}
$$

where $i=1,2, \ldots, K$.

(b) Calculation of the gains for each initial precoding vector:

$$
\mathrm{G}_{p, k, i, n}=\left\|\mathbf{W}_{p, i, n}^{\text {init }} \mathbf{H}_{p, k, n}\right\|^{2}
$$

(c) Calculation of supportable bits for each initial precoding vector.

$$
c_{p, k, i, n}=\min \left(f^{-1}\left(\frac{P_{T}}{N} \mathrm{G}_{p, k, i, n}\right), M\right)
$$

(d) Selection of both the precoding vector and the user that maximize the sum data rate:

Calculate the tentative total data rate $\bar{R}_{p, k, i, n}$ when the $k$ th user is selected as the user requiring the maximum power for each initial precoding vector.

$\bar{R}_{p, k, i, n}=u_{p, k, i, n} c_{p, k, i, n}$

where $u_{p, k, i, n}$ indicates the number of users who have channel gains larger than $\mathrm{G}_{p, k, i, n}$.

(e) Select the user index $\kappa$ and the precoding vector index $\varrho$ maximizing $\bar{R}_{p, k, i, n}$.

$\kappa, \varrho=\arg \max _{k, i} \bar{R}_{p, k, i, n}$

Then, the precoding vector is chosen as

$\mathbf{W}_{p, n}=\mathbf{W}_{p, Q, n}^{\text {init }}$

(f) Allocation of the users to the subcarrier:

$\rho_{p, k, n}= \begin{cases}1 & \text { if } \quad \mathrm{G}_{p, k, \varrho, n} \geq \mathrm{G}_{p, \kappa, \varrho, n} \\ 0 & \text { else }\end{cases}$

Step 2mp) Bit loading:

The same algorithm as given in Step $2 s w$ by using $\mathrm{G}_{p, \kappa, \ell, n}$ instead of $H_{p, w_{n}, n}$. 
Fig. 3 The transmission chain for multicast MISO-OFDM systems

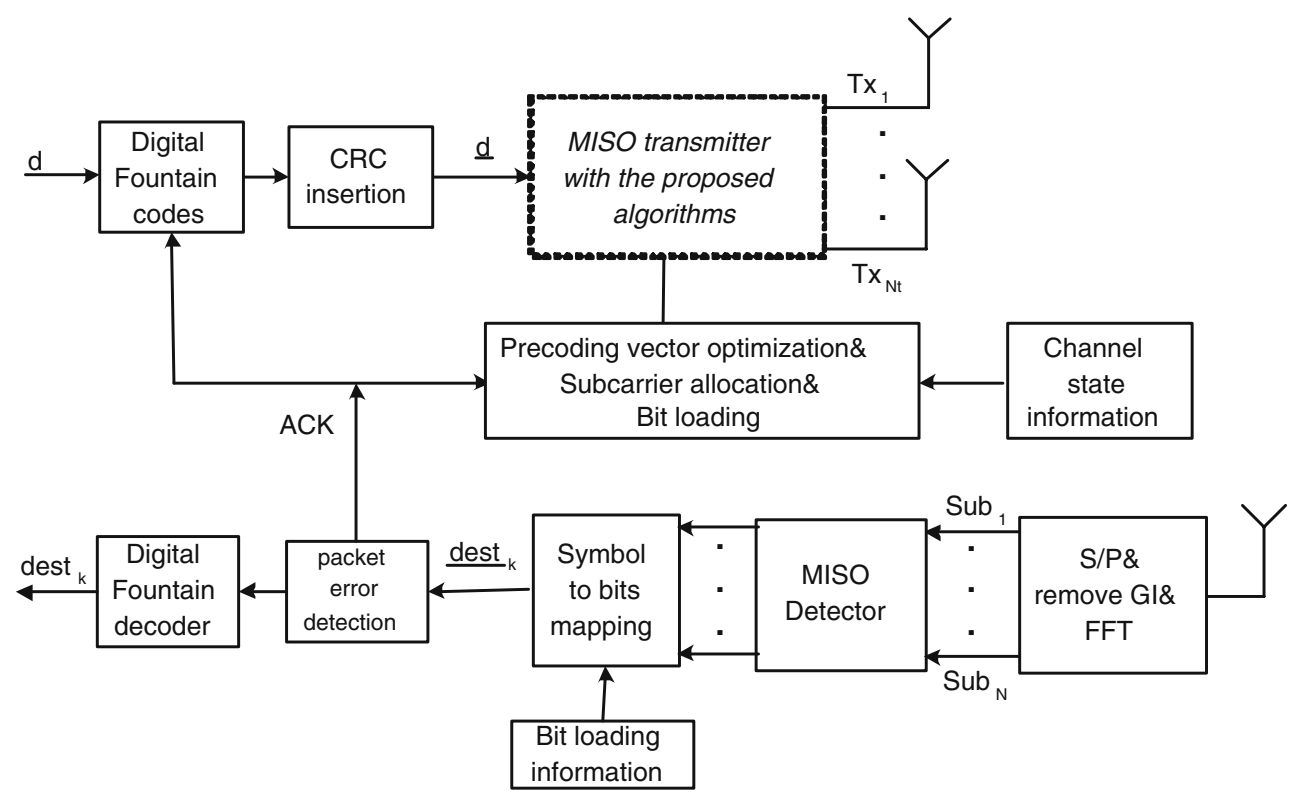

\section{General description of the proposed transmission chain}

The original source data is first divided in superframes composed of $K_{p}$ packets of $K_{l}$ bits. There packets are encoded, transmitted and the users can reconstruct the original source data once they have received a sufficient number of encoded packets. According to the capacity of the erasure channels, $K_{p}^{\prime}$ correctly received packets should be theoretically sufficient to decode the superframe of $K_{p}$ packets, assuming that $K_{p}$ is long enough. Fountain codes have efficient encoding and decoding algorithms and allow the recovery of the original superframe from any $K_{p}^{\prime}$ encoded packet with high probability, where $K_{p}^{\prime}$ is just slightly larger than $K_{p}$. In this work, we will use LT codes [10] that are particularly well adapted to multicast systems because they can generate encoded packets until all the users have correctly received at least $K_{p}^{\prime}$ encoded packets. The main benefit of this approach is that different receivers can recover the source data using different encoded packets. When a user has received $K_{p}^{\prime}$ packets, it sends an acknowledge (ACK) to the transmitter and, consequently, the subcarrier allocation eliminates this user from the set of the receivers until the end of the superframe.

The LT codes can be seen as irregular low-density generator matrix codes. Each encoded packet is generated by performing the bitwise modulo 2 sum of $d_{n}$ source packets. The degree $d_{n}$ is chosen from a degree distribution $\rho(d)$. In this paper, we have considered the robust soliton distribution to avoid failure of the decoding [10]. In addition, each encoded packet is labeled and a cyclic redundancy check (CRC) is added. For this chain, it is not necessary to send subcarrier information to the users.

At the end of transmission where all users receives $K_{p}^{\prime}$ packets, the supportable data rate by the transmission chain is calculated by:

$\mathrm{DR}=\frac{K_{p} \times K_{l}}{T_{O F D M} \times P_{f} \times F}$

where $K_{l}$ is the number of bits per packet, $F$ is the number of necessary frames to send enough packets to all users, $T_{O F D M}$ is the duration of the OFDM symbol, and $P_{f}$ is the number of OFDM symbols in one frame. We assume that the channel coefficients remain constant during one frame.

The transmission chain for the multicast MISOOFDM systems is shown in Fig. 3. The same transmitter and receiver scheme is used for SISO-OFDM systems by setting $N_{t}=1$, using the proposed SISO algorithm at the transmitter, the SISO detector at the receiver and discarding the precoding vector optimization part. This transmission chain will be used to evaluate the average user data rates in the next section.

\section{Simulation results}

In this section, we perform the simulation results using the Hiperlan/2 standard [15] and the channel model A [16], which corresponds to a typical office environment with 9 channel taps. The total OFDM symbol duration 


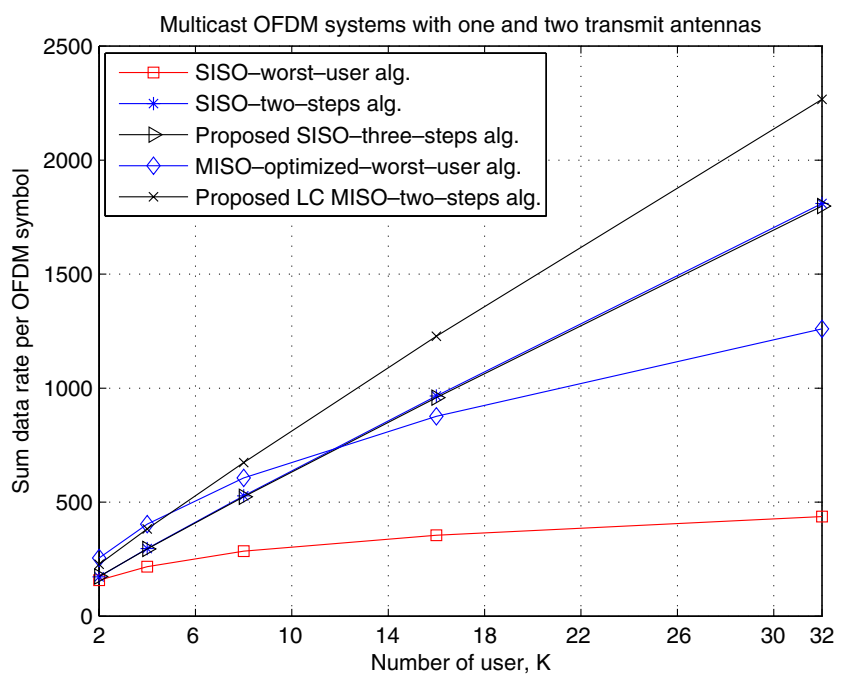

Fig. 4 The average sum data rate results for multicast SISO/MISO-OFDM systems with $N_{t}=2$ antennas

is $4 \mu \mathrm{s}$, including a $0.8-\mu \mathrm{s}$ guard interval. It consists of $N=64$ subcarriers. The Doppler frequency is set at $50 \mathrm{~Hz}$. The required BER, the noise variance, and the number of bits per symbol are chosen as $p_{e}=10^{-4}$, $N_{0}=1$, and $M=1,2,4,8$, respectively. The transmitted power is fixed at $P_{T}=30 \mathrm{dBW}$. The average data rates are calculated for one OFDM symbol to obtain results independent from the frame length.

In the first part, we evaluate the average sum data rate as defined in Eq. 2 and the average minimum user data rate using the schemes in Figs. 1 and 2. The number of frames is fixed to $P=100$ and the number of OFDM symbols per frame is $P_{f}=256$ according to the channel time variation.

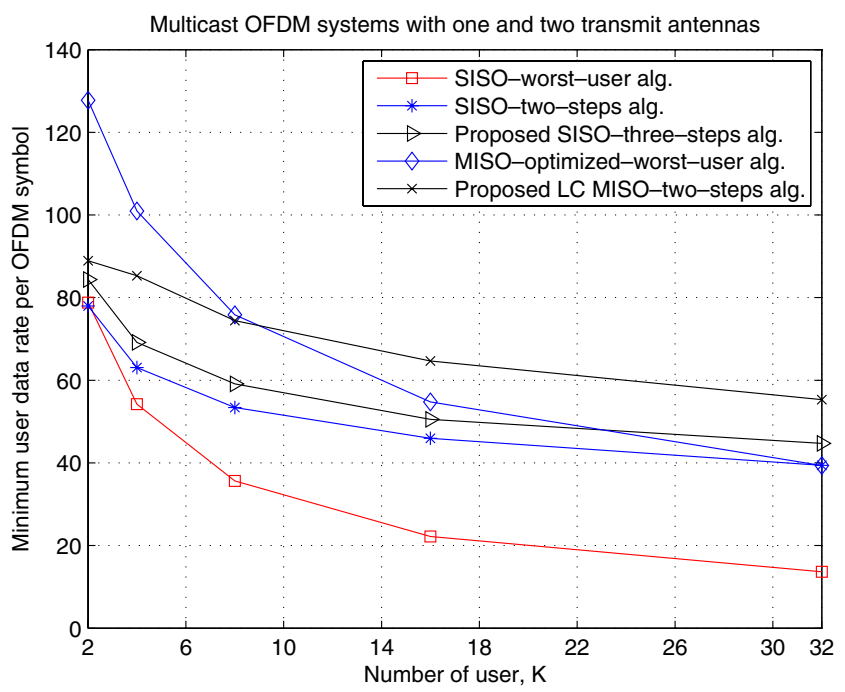

Fig. 5 The average minimum user data rate results for multicast SISO/MISO-OFDM systems with $N_{t}=2$ antennas

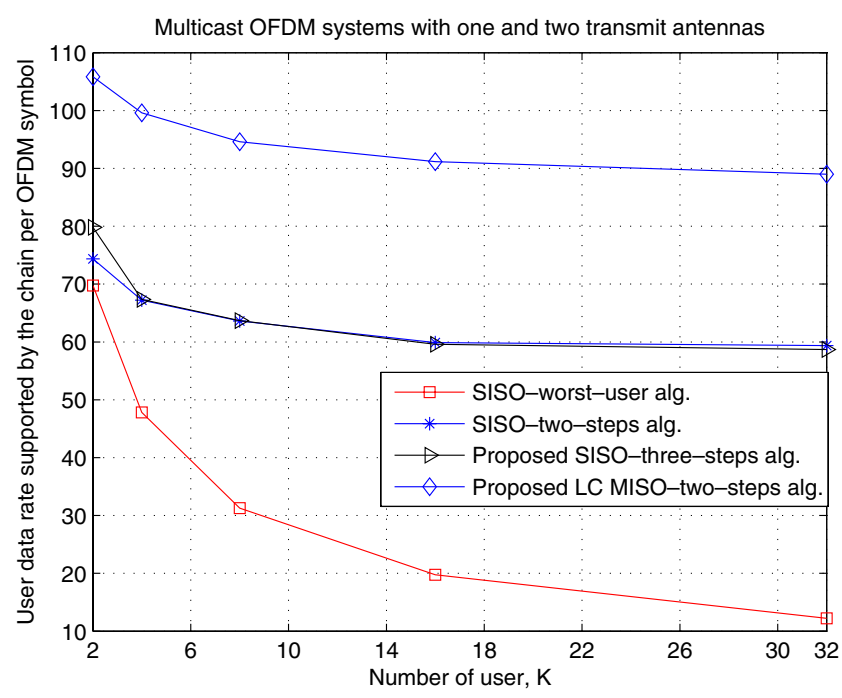

Fig. 6 The average user data rate results supported by the proposed transmission chain for multicast SISO/MISO-OFDM systems with $N_{t}=2$ antennas

As given in Fig. 4, the average sum data rate results of both the SISO-two-steps and the proposed SISOthree-steps algorithms increase almost linearly with the number of users compared to the SISO-worst-user algorithm. Besides, it is shown that the proposed MISOtwo-steps algorithm increases the average sum data rate compared to SISO-worst-user and MISO-optimizedworst-user algorithms. The average minimum user data rate results given in Fig. 5 show that, for both single and multiple transmit antenna cases, the two-step algorithm outperforms the worst-user algorithm. For MISO-OFDM, the proposed two-steps algorithm

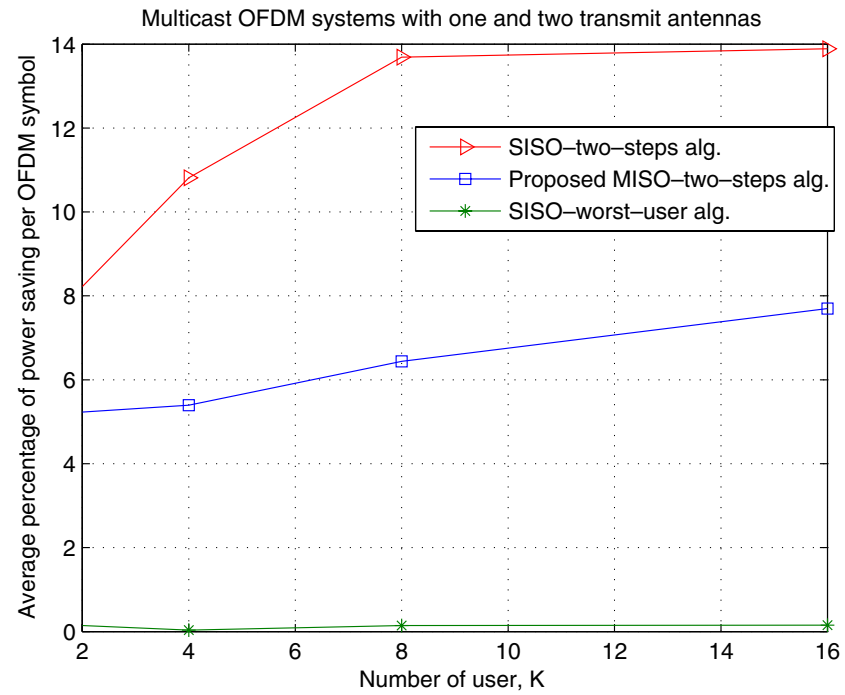

Fig. 7 The average percentage of power saving of the proposed SISO/MISO-OFDM algorithms compared to worst-user case for the proposed transmission chain 
provides the higher minimum user data rate performance when the number of users is higher than 8 . Furthermore, the proposed algorithm is less complex than the optimized worst user algorithm, which implies a max-min optimization procedure.

In the second part, we evaluate the average user data rate results supported by the proposed transmission scheme as defined in Eq. 50 for the scheme given in Fig. 3. The packet size and the number of packets of the superframe are fixed at $K_{l}=128$ bits and $K_{p}=$ 10000 , respectively. To perfectly decode the transmit data thanks to the LT code properties, we choose $K_{p}^{\prime}=$ 11000. A 16-bit CRC is applied to each encoded packet and ACK is transmitted by the users when they receive $K_{p}^{\prime}$ packets. The user will then switch into a sleep mode to reduce power consumption. We assume that the CSI is updated every $P_{f}=256$ OFDM symbols. At the receiver, only modulation indexes for each subcarrier are required and maximum likelihood detector at subcarrier level is performed by assuming that the channel has been perfectly estimated using pilots.

According to the performance results given in Fig. 6, the SISO-two-steps and the proposed MISO-two-steps algorithms provide a higher average user data rate than the worst-user algorithms for the proposed transmission chain. These results are coherent with the average minimum user data rate results obtained in Fig. 5.

Another important issue concerning the resource allocation algorithm is the power saving [17]. Indeed, when a user has received enough packets, it sends back an ACK to the transmitter and switches into a sleep mode. As shown in Fig. 7, the proposed algorithms for SISO and MISO case provide, respectively, $8 \%$ and $14 \%$ of power saving for $K=16$ users compared to the worst user algorithms.

\section{Conclusion}

In this work, we have evaluated the performance of multicast OFDM systems with single and multiple transmit antennas considering different resource allocation algorithms. Since the optimal algorithms that include the subcarrier allocation and bit loading and precoding vector selection in the MISO case are too complex, we proposed different suboptimal algorithms based on the maximization of the sum data rate or the maximization of the minimum user data rate. We have shown that the SISO-three-steps algorithm slightly increases the minimum user data rate compared to the SISO-two-steps algorithm that maximizes the sum data rate. The proposed algorithm also outperforms the worst user case algorithm. We have shown that it is possible to further increase the sum data rate and minimum user data rate using multiple transmit antennas. We have proposed suboptimal algorithms that are able to extract the gain of multiple transmit antennas while avoiding complex optimization. We have shown that the proposed two-step algorithm significantly improves the performance compared to the worst case algorithm.

We have also considered a complete transmission chain for practical application by combining powerful erasure codes with the previously proposed algorithms for both single and multiple transmit antennas. The obtained average user data rate results confirm that the proposed algorithms provide better performance for both single and multiple transmit antennas than worst user case. Another important aspect of the resource allocation algorithm is power saving. We have shown that $10 \%$ of the total power consumption can be saved by using the proposed algorithms compared to the worst user algorithms.

These algorithms have been developed and performed at a subcarrier level. However, they can also be applied to subcarrier clusters to reduce the feedback load. It will be also interesting to extend these results by evaluating the impact of the feedback and quantization errors on the performance of the proposed algorithms.

\section{References}

1. Varshney U (2002) Multicast over wireless networks. Commun ACM 45:31-37 (December)

2. Cimini L (1985) Analysis and simulation of a digital mobile channel using orthogonal frequency multiplexing. IEEE Trans Commun 33:665-675

3. Foschini GJ, Gans MJ (1998) On the limits of wireless communications in fading environment when using multiple antennas. Wirel Pers Commun 6:311-335

4. Bolcskei H, Gesbert D, Paulraj AJ (2000) On the capacity of OFDM-based multi-antenna systems. Proc IEEE Int Conf Acoust Speech Signal Proc 5:2569-2572

5. Wong CY, Cheng RS, Ben Letaief K, Murch RD (1999) Multiuser OFDM with adaptive subcarrier bit and power allocation. IEEE J Sel Areas Commun 17:1747-1758 (October)

6. Shen Z, Andrews JG, Evans BL (2003) Optimal power allocation in multiuser OFDM systems. In: Proc of IEEE Globecom conference. IEEE, Piscataway, pp 337-341 (December)

7. Suh C, Hwang CS (2004) Dynamic subchannel and bit allocation for multicast OFDM Systems. In: Proc of IEEE PIMRC, Barcelona, September 2004

8. Özbek B, Le Ruyet D, Khiari H (2006) Adaptive resource allocation for multicast OFDM systems with multiple transmit antennas. In: Proc of the IEEE ICC. Istanbul, June 2006 
9. Le Ruyet D, Özbek B, Khiari H (2007) Performance of multicast MISO-OFDM systems. In: Proc of 13th European wireless conference, Paris, April 2007

10. Luby M (2002) LT codes. In: Proc of 43rd annual IEEE symposium on foundations of computer science (FOCS), Vancouver, 16-19 November 2002

11. Byers JW, Luby M, Mitzenmacher M (2002) A digital fountain approach to asynchronous reliable multicast. IEEE J Sel Areas Commun 20:1528-1540 (October)

12. Shokrollahi A (2006) Raptor codes. IEEE Trans Inf Theory 52:2551-2567

13. Proakis JG (1995) Digital communication, 3rd edn. McGraw Hill, New York
14. Sidiropoulos ND, Davidson TN, Luo ZQ (2006) Transmit beamforming for physical layer multicasting. IEEE Trans Signal Proc 54:2239-2251 (June)

15. Khun-Jush J, Schramm P, Wachsmann U, Wenger F (1999) Structure and performance of the hiperlan/2 physical layer. In: Proc of the IEEE VTC, vol 5. IEEE, Amsterdam, pp 2667-2671 (June)

16. ETSI Normalization Committee (1998) Channel models for hiperlan/2 in different indoor scenarios. ETSI, SophiaAntipolis

17. Xiao Y (2005) Energy saving mechnism in the IEEE 802.16e wireless MAN. IEEE Commun Lett 09:595-597 (July) 